\title{
Tandem Light-Guides With Micro-Line-Prism Arrays for Field-Sequential-Color Scanning Backlight Module
}

\author{
Chung-Hao Tien, Yen-Hsing Lu, and Yuan-Jung Yao
}

\begin{abstract}
An optically partitioned backlight system consisting of tandem wedge-shaped light-guides (LGs) with micro-line-prism arrays was developed for scanning field-sequential-color (FSC) liquid crystal display (LCD) in large size. The unit of wedge-shaped LG combined with a light-emitting diode (LED) light-bar was designed and fabricated to collimate the extraction light within a narrow angular extent. Based on the edge lighting approach, the volume of backlight system can be reduced down to $25 \mathrm{~mm}$ without sacrifice of the optical behavior. As a result, 2750 nits average luminance subject to $50 \%$ duty cycle and $83 \%$ uniformity can be achieved. The leakages into the consecutive adjacent blocks were well suppressed to $11.86 \%$.
\end{abstract}

Index Terms-Field-sequential-color (FSC) liquid crystal display (LCD), micro-line-prism arrays, scanning backlight, tandem light-guides (LGs).

\section{INTRODUCTION}

$\mathbf{H}$ IGHLY COLORED images rendered via liquid crystal displays (LCDs) can be accomplished based on the trichromatic approach in either spatial or temporal arrangement. The former approach yields three primary RGB colors by means of color filters (CFs) which are coated in the exitance side of the LC cells [1]-[3], [18]. However, only one third of light from back lighting is allowed to pass through the CFs with the limited spectral transmission and result in a huge loss in terms of energy saving. Therefore, CF-free LCDs associated with temporally color sequential driving has increasingly been the subject during the recent years [4]-[8]. Field-sequential-color (FSC) system utilizes the fast optically-compensated-bend (OCB) mode LC and light-emitting diodes (LEDs) to implement a fast switching response. Three primary RGB colors were serially present over the full screen during each frame

Manuscript received December 20, 2007. This work was supported by the Ministry of Economic Affair, Technology Development Program for Academia, Taiwan, R.O.C., under Contract 96-EC-17-A-07-S1-046 and by the National Science Council, Taiwan, R.O.C., under Contract NSC 96-2221-E-009-114MY3.

C.-H. Tien and Y.-H. Lu are with the Department of Photonics and Display Institute, National Chiao Tung University, Hsinchu 300, Taiwan, R.O.C. (e-mail: chtien@mail.nctu.edu.tw)

Y.-J. Yao was with Department of Photonics and Display Institute, National Chiao Tung University, Hsinchu 300, Taiwan, R.O.C.. He is now with AUOptronics, Hsinchu Science Park, Hsinchu, 30078, Taiwan, R.O.C.

Color versions of one or more of the figures in this paper are available online at http://ieeexplore.ieee.org.

Digital Object Identifier 10.1109/JDT.2008.917090

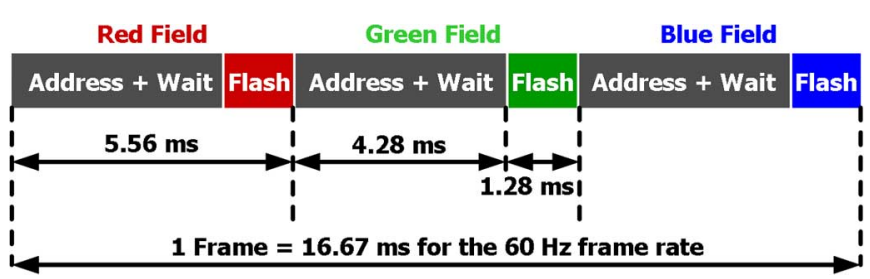

Fig. 1. Time chart of a practical 32 -in sequential-color LCD with a $60-\mathrm{Hz}$ frame rate. Time of LED lighting only occupied $23 \%$ in each subfield.

time; thus, the full color image can be recognized due to the visual residue.

An adequate duration including the thin-film-transistor (TFT) access time, LC response, and LED flashing is essential to exhibit a correct picture. Taking a 32-in FSC LCD (Fig. 1) as example [9], the frame rate is at least $60 \mathrm{~Hz}$ to alleviate the flickering effect. Each subframe allows only $4.28 \mathrm{~ms}$ on data writing and LC response accordingly. After TFT arrays and LC were refreshed and saturated in each subframe, the LEDs with single color are illuminated and turned off before the start of the next subframe. Accordingly, the optical characteristics of LC panel such as LC response time and TFT addressing have a huge impact upon the duty cycle of LED flashing. Such issues would be more concerned in the large size.

As illustrated in Fig. 1, time of LED lighting only occupied 23\% in each subfield [9]. The insufficient brightness caused by short flashing can be overcome by using overdriving LED current, as the way adopted in cathode-ray tube (CRT). Nonetheless, another undesirable performance like incorrect color mixing and motion blur still occurs due to the slow LC response.

In this study, we attempted to develop a novel optical backlight system sequentially operated in both spatial and temporal arrangement to spare more LED duration and thus alleviate above undesirable issues [9]-[14]. A 32-in optomechanical setup was partitioned into $12 \times 4$ tandem wedge-shaped light-guides (LGs) associated with the edge lighting. In addition to keep the merits of the brightness and uniformity, the proposed feature would lead to a more compact volume compared with the typical direct-view approach in large FSC LCDs. The advantage of modularization can be scaled up to any other size by modifying the relevant parameters of each mosaic structure accordingly. 


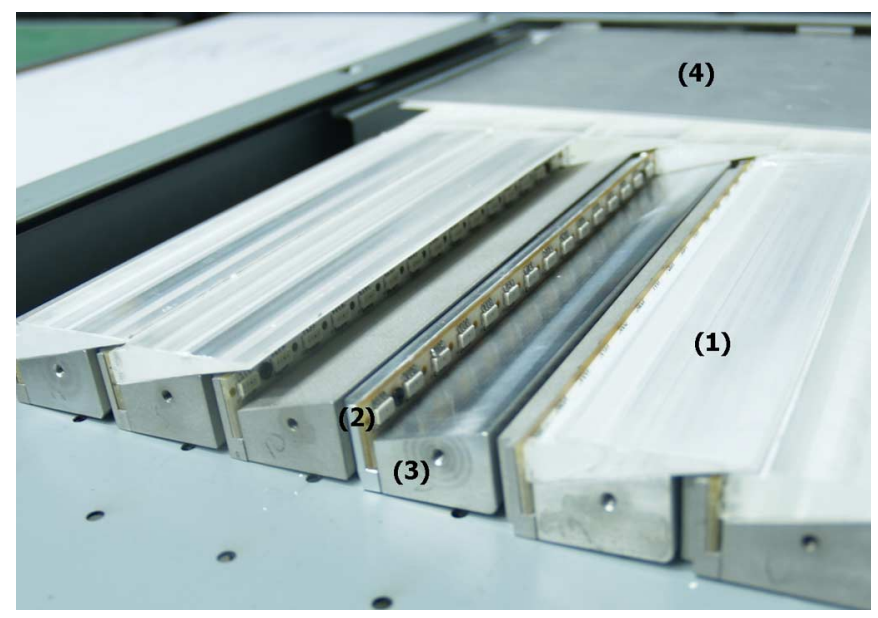

Fig. 2. Configuration of proposed scanning backlight module which consists of: (1) wedge-shape light guides; (2) LED light-bars; (3) optomechanical holder; (4) diffusing and prism sheets.

\section{TANDEM LG FOR LARGE-SIZED FSC SCANNING BACKLIGHT MODULE}

\section{A. Backlight Configuration}

The configuration of proposed scanning backlight module (BLM) is shown in Fig. 2. The backlight consists of the following.

1) Wedge-shape light guides were arranged in a tandem configuration. Each LG accompanied a micro-line-prism array over the back surface to control the emitting luminance distribution toward the diffusing and prism sheet upon the LGP. In order to increase the extraction efficiency, high-reflective aluminum was coated on the bottom surface of each wedge-shape LG unit,

2) LED light-bars were set in front of the entrance plane of the corresponding LG and beneath the prior one. Each light-bar had 15 packages of full color LEDs, and each package contained four chips of RGGB subject to the dominant wavelength $\lambda_{\mathrm{R}}=624 \mathrm{~nm}, \lambda_{\mathrm{G}}=530 \mathrm{~nm}$, and $\lambda_{\mathrm{B}}=464 \mathrm{~nm}$, respectively [15],

3) Optomechanical holder made of aluminum was both functioned as the thermal conducting channel and LG constitution,

4) Diffusing and prism sheets were employed to increase uniformity and convert the light direction toward the surface normal.

The twelve vertical partitions were set to operate in dual scan with 50\% duty cycle, as shown in Fig. 3(a) [11]. Meanwhile, the four horizontal divisions were used for the two dimensional scanning approach, as shown in Fig. 3(b) [14]. Each LG unit has the width and length of 37.2 and $177.1 \mathrm{~mm}$, respectively. Detailed multi-area scanning and sequential algorithm can refer to other publications [11]-[14].

\section{B. Optical Design}

The proposed wedge-shaped LG unit is based on the reflection-refraction characteristics of the prismatic pattern with modulated pitches on the bottom surface. In order to preserve the adequate space for light-bar constitution, the included angle

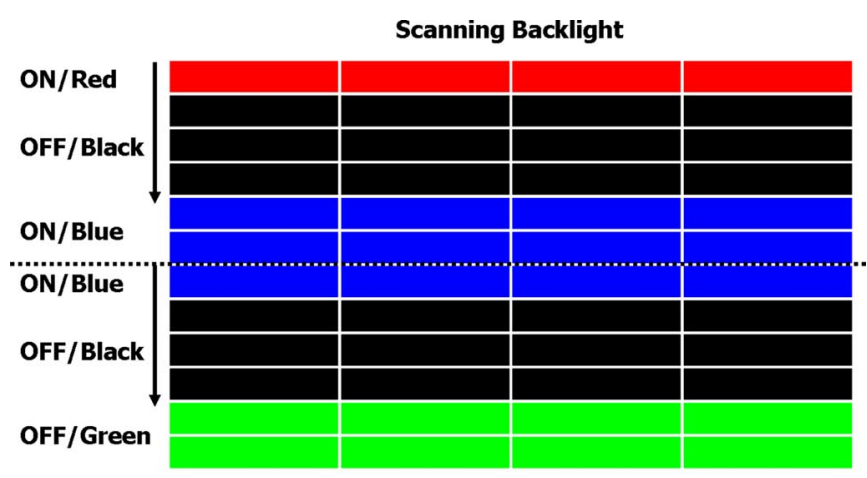

(a)

Sub-frame b1

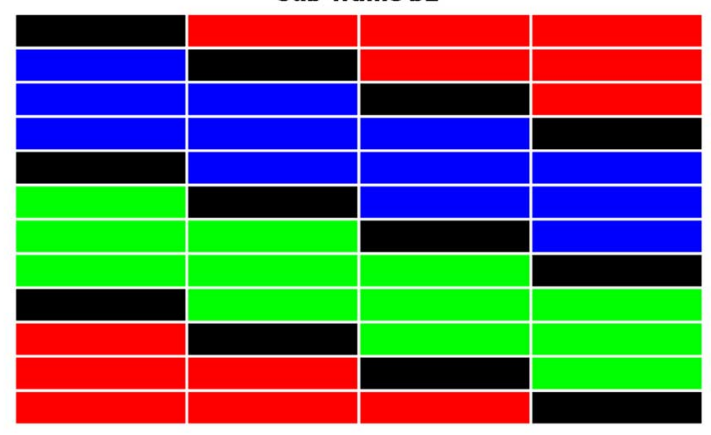

(b)

Fig. 3. Two graphics examples of multi-area scanning and sequential algorithm: (a) 12 vertical partitions are set to operate in dual scan with 50\% duty cycle [11] and (b) the four horizontal divisions are used for the 2-D scanning approach [14].

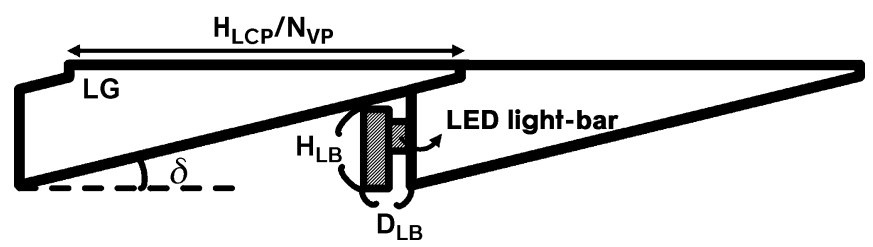

Fig. 4. Schematic relations between the included angle $(\delta)$ and light-bar constitution.

$(\delta)$ subtended to the height of the light-bar $\left(\mathrm{H}_{\mathrm{LB}}\right)$ is determined by using triangular relations as follows:

$$
\delta=\tan ^{-1}\left(\frac{\mathrm{H}_{\mathrm{LB}}}{\left(\mathrm{H}_{\mathrm{LCP}} / \mathrm{N}_{\mathrm{VP}}\right)-\mathrm{D}_{\mathrm{LB}}}\right)
$$

where $\mathrm{H}_{\mathrm{LCP}}$ is the height of LC panel, $\mathrm{N}_{\mathrm{VP}}$ is the numbers of the vertical partitions, and $\mathrm{D}_{\mathrm{LB}}$ is the thickness of the light-bar as illustrated in Fig. 4. For the case of 32-in BLM with 50\% duration in dual scan, the angle $\delta$ is defined as $13.6^{\circ}\left(\mathrm{N}_{\mathrm{VP}}=\right.$ $12, \mathrm{H}_{\mathrm{LB}}=8.15 \mathrm{~mm}$, and $\mathrm{D}_{\mathrm{LB}}=3.5 \mathrm{~mm}$ ). Each unit should be well confine to avoid the color mixing around the boundary between individual cells. A certain amount of light leakage to the adjacent unit would lead to undesirable image distortion and incorrect color mixture.

In order to obtain the uniform and directional luminance, the micro-line-prism arrays were fabricated over the back surface of the LG by the diamond turning method. Rays from each RGB chip can be coupled into the LG and mixed after multiple reflections by the designed micro bumps. The dependence of optical 


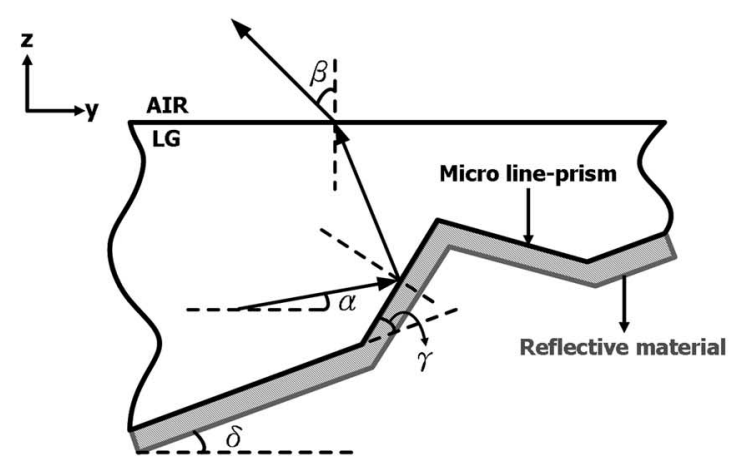

(a)

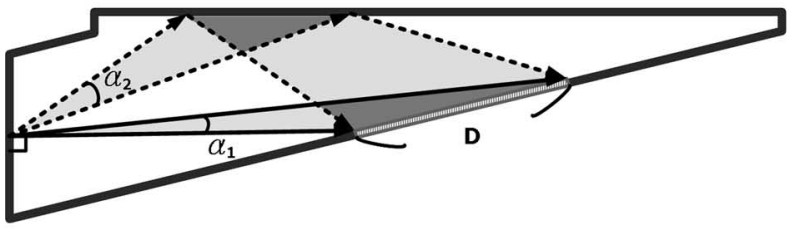

(c)

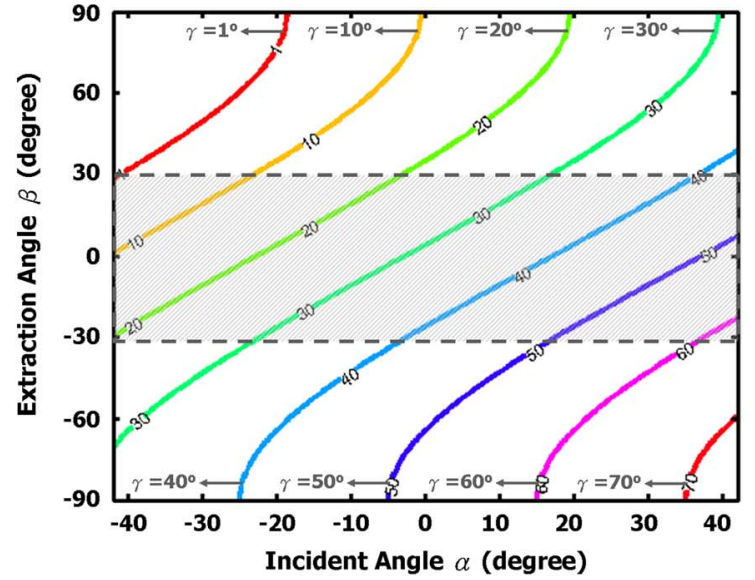

(b)

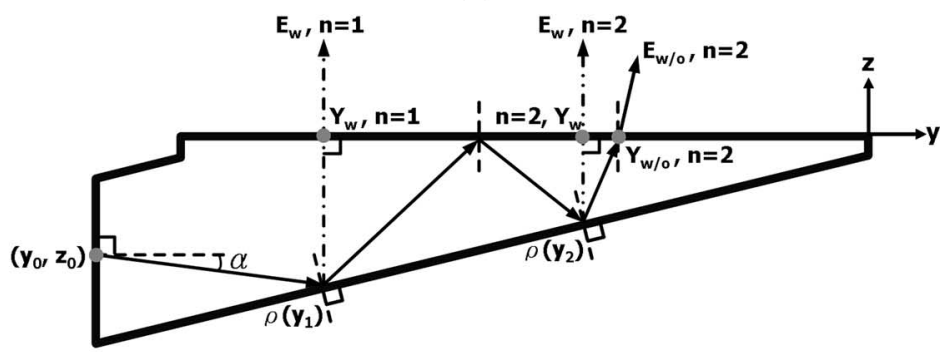

(d)

Fig. 5. (a) Principle of optical coupling behavior in the wedge-shape light-guide. (b) Extraction angles achieved by (2) for different orientations of the reflective micro taper prisms. The gray region reveals a proper operated range to confine the luminance divergence. (c) Incident rays with half cone angle $\alpha_{1}=0^{\circ} \sim 5^{\circ}$ and $\alpha_{2}=15^{\circ} \sim 20^{\circ}$ lay on the target region (D). Referring to the Fig. 4(b), the angular distribution at base angle $\gamma=32^{\circ}$ in D has the narrowest full width at half maximum about $\pm 15^{\circ}$; (d) the behavior of extracting light is controlled by either the inclined bottom surface or the micro-line-prisms. In the case of this figure, the incident angle $\alpha<\tan ^{-1}\left(y_{0} / z_{0}\right)$ and $m=2$.

path on the geometric setup is illustrated in Fig. 5(a). For simplicity, each LED was assumed to be set in the centre of incident plane with Lambertian angular radiation pattern. Based on Snell's Law, the extraction angle $\beta$ can be expressed as a function of the incident angle $(\alpha)$, inclination of the wedge $\operatorname{LG}(\delta)$, and the base angle of prism $(\gamma)$ as

$$
\beta=\sin ^{-1}\left(\mathrm{n}_{\mathrm{LG}} \sin \left(\alpha+90^{\circ}-2(\gamma+\delta)\right)\right)
$$

where $n_{L G}$ is the refractive index of light guide. For PMMA material with $\mathrm{n}_{\mathrm{LG}}=1.49$, the cone angle $(\alpha)$ from the LED radiant flux was within $\pm 42^{\circ}$. According to the interval of incident angle with different base angle, the corresponding extraction angle can be shown in Fig. 5(b). The gray region reveals a proper operated range to confine the luminance divergence. Consequently, every position over the oblique surface can determine a coincident $\gamma$ value to ensure the emanating light with a narrow angular distribution. Taking a graphic solution for the case in Fig. 5(c), the incident rays subject to half cone angle $\alpha_{1}=0^{\circ}-5^{\circ}$ and $\alpha_{2}=15^{\circ}-20^{\circ}$ would lie on the target region (D). Referring to the Fig. 5(b), an adequate base angle is around $30^{\circ}-36^{\circ}$. The angular distribution at base angle $\gamma=32^{\circ}$ had the narrowest full-width at half-maximum (FWHM) about $\pm 15^{\circ}$. By means of the same rules, the suitable $\gamma$ of the other position over the incline surface can be obtained by a range of $30^{\circ}-40^{\circ}$ accordingly.
In order to increase the uniformity of the LGP, an optimum layout was performed with modulated pitch based on the radiometry, geometric ray tracing, and probability density function. Radiant flux from light source could be divided into any number of angular regions with equal energy. According to energy conservation theory, the $i_{\text {th }}$ angular interval of Nregions follows

$\sin ^{-1}\left[\frac{1}{n_{\mathrm{LG}}} \sin \left(\frac{i-1}{N}\right)\right] \leq \theta_{i t h}<\sin ^{-1}\left[\frac{1}{n_{\mathrm{LG}}} \sin \left(\frac{i}{N}\right)\right]$

Hence, the illuminance was employed to evaluate the uniform quality over the extracting surface by means of the identical flux unit and ray tracing principle. The behavior of extracting light was constrained by either the inclined bottom surface or the micro-line-prisms as illustrated in Fig. 5(d). The relationship between incident angle $(\alpha)$ and emergent position (Y) with (w) or without (w/o) the influence of taper prisms can be deduced as

$$
Y_{\mathrm{w}}=\left\{\begin{array}{cc}
\frac{-z_{0}+y_{0} \tan \alpha}{\tan \alpha+\tan (2 n \delta)}, & \alpha \geq \tan ^{-1}\left(\frac{y_{0}}{z_{0}}\right), \\
\frac{z_{0}+y_{0} \tan |\alpha|}{\tan |\alpha|+\tan [(2 n-1) \delta]}, & \quad x<1,2, \ldots, m-1 \\
& n=1,2, \ldots, m
\end{array}\right.
$$

$Y_{\mathrm{w} / \mathrm{o}}=\sqrt{y^{2}+z^{2}}$ 


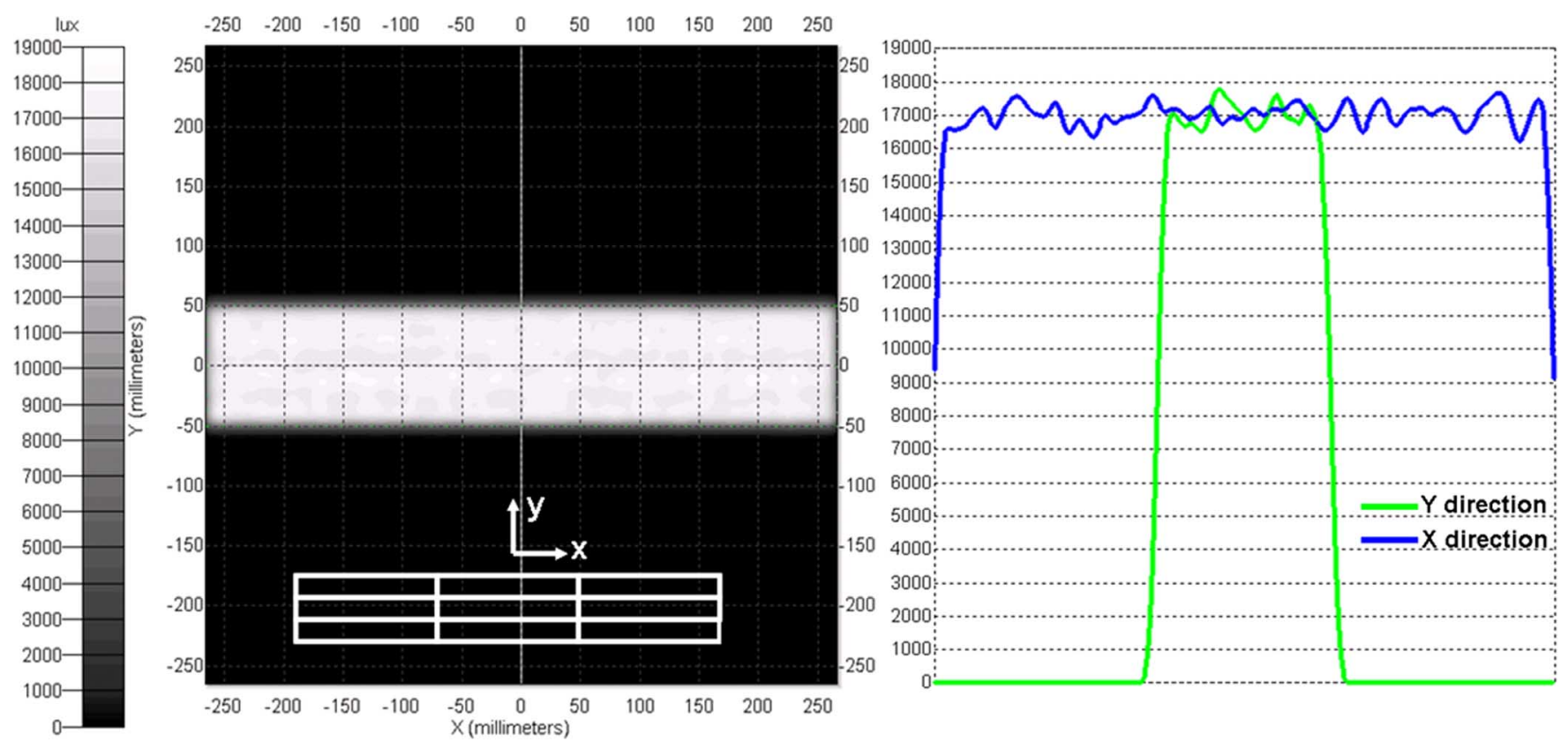

Fig. 6. Luminance distribution of a $3 \times 3$ partitions optimized by optical software TracePro. No indistinguishable discontinuity is around the boundary.

where

$$
\begin{aligned}
& y= \begin{cases}\frac{-z_{0}+y_{0} \tan \alpha}{\tan \alpha+\tan [2(m-1) \delta]}, & \alpha \geq \tan ^{-1}\left(\frac{y_{0}}{z_{0}}\right) \\
\frac{z_{0}+y_{0} \tan |\alpha|}{\tan |\alpha|+\tan (2 m \delta)}, & \alpha<\tan ^{-1}\left(\frac{y_{0}}{z_{0}}\right)\end{cases} \\
& z= \begin{cases}z_{0}+\left(y-y_{0}\right) \tan \alpha, & \alpha \geq \tan ^{-1}\left(\frac{y_{0}}{z_{0}}\right) \\
z_{0}-\left(y-y_{0}\right) \tan |\alpha|, & \alpha<\tan ^{-1}\left(\frac{y_{0}}{z_{0}}\right) .\end{cases}
\end{aligned}
$$

The coordinates $\left(y_{0}, z_{0}\right)$ in (4) present the position of the LED. The variable $m$ indicates that the light exited outside LG after reaching the extracting surface at the $m_{\mathrm{th}}$ time and is given as

$$
m= \begin{cases}\operatorname{ceiling}\left(\frac{90^{\circ}-\alpha-\theta_{c}}{\delta}\right)+1, & \alpha \geq \tan ^{-1}\left(\frac{y_{0}}{z_{0}}\right) \\ \operatorname{ceiling}\left(\frac{90^{\circ}-\alpha-\theta_{c}}{2 \delta}\right), & \alpha<\tan ^{-1}\left(\frac{y_{0}}{z_{0}}\right)\end{cases}
$$

where the ceiling function gives the nearest integer value larger than the exact value [16], and $\theta_{\mathrm{c}}$ is the critical angle based on the Snell's law. Besides, we assume that a skew ray propagated toward the normal direction after reflecting by micro structures for simplicity. Referring to (4) and (5), the coincide illuminances at the specific position Y according as the unit flux is

$$
\begin{aligned}
E_{\mathrm{w}}= & \sum_{i=1}^{n}\left[(-1)^{n+i} \prod_{i}^{n} \rho\left(y_{i}\right)\right], \\
& \begin{cases}\alpha \geq \tan ^{-1}\left(\frac{y_{0}}{z_{0}}\right), & n=1,2, \ldots, m-1 \\
\alpha<\tan ^{-1}\left(\frac{y_{0}}{z_{0}}\right), & n=1,2, \ldots, m\end{cases} \\
E_{\mathrm{w} / \mathrm{o}}=1- & \sum_{i=1}^{n}\left[(-1)^{n+i} \prod_{i}^{n} \rho\left(y_{i}\right)\right], \\
& \begin{cases}\alpha \geq \tan ^{-1}\left(\frac{y_{0}}{z_{0}}\right), & n=m-1 \\
\alpha<\tan ^{-1}\left(\frac{y_{0}}{z_{0}}\right), & n=m\end{cases}
\end{aligned}
$$

In (9) and (10), $\rho(\mathrm{y})$ is a controllable parameter, fill factor, which was introduced to determine the optical reflection are

$$
\rho(y)=\frac{W(y)}{P(y)}
$$

where $W(y)$ and $P(y)$ represent the width and pitch of microline-prism, respectively. In other words, $\rho(\mathrm{y})$ can be regarded as a probability density function used to estimate whether the guiding light reached the taper prism or not. The combination of prism density and taper angle would maximize the uniformity at an appropriate angular extent for each partitioned unit. The variation of illuminance shall be kept under $\pm 10 \%$ average value to ensure at least $82 \%$ uniformity. Under the fill factor ranging from $16 \%$ to $68 \%$, the calculated uniformity and optical efficiency were about $93 \%$ and $68 \%$, respectively. Fig. 6 illustrates the luminance distribution of a $3 \times 3$ partitions. Indistinguishable discontinuity around the boundary and $9.2 \%$ leakage into adjacent blocks firstly confirm the optical characteristics of the designed LG.

\section{RESULTS AND DISCUSSION}

Luminance in angular distribution of the experimental LGP with diffusing and prism sheets was measured in Fig. 7. The $z$-axis is assumed to be the surface normal. The azimuthal and zenith angles in polar coordinates are defined as $\phi=0^{\circ}-360^{\circ}$ and $\theta=0^{\circ}-90^{\circ}$, respectively. Since the taper-shaped line prisms were functioned to redirect the emergent angle along the azimuthal directions $(\phi)$ of $90^{\circ}$ and $270^{\circ}$, only single prism sheet (BEF) was required to convert the azimuth direction. The FWHM of the luminance was an elliptical angular extent with $\theta_{\mathrm{x}}$ (major axis) $=37^{\circ}$ and $\theta_{\mathrm{y}}$ (minor axis) $=30^{\circ}$, respectively. Highly directional luminance associated with limited zenith angle $(\theta)$ provided the benefit against the color mixture afterward. 


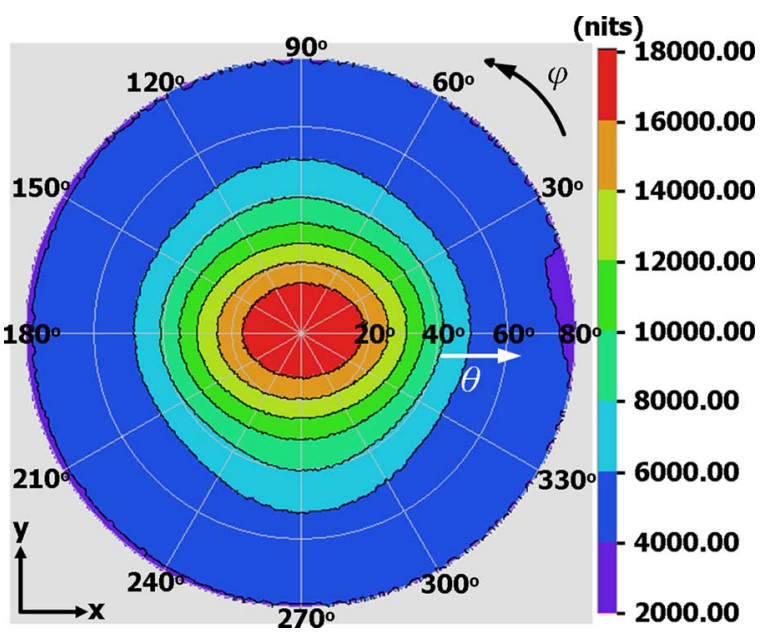

Fig. 7. Luminance in angular distribution of the experimental light-guide plate with diffusing and prism sheets. The FWHM of the luminance was an elliptical angular extent with $\theta_{\mathrm{x}}=37^{\circ}$ and $\theta_{\mathrm{y}}=30^{\circ}$, respectively.

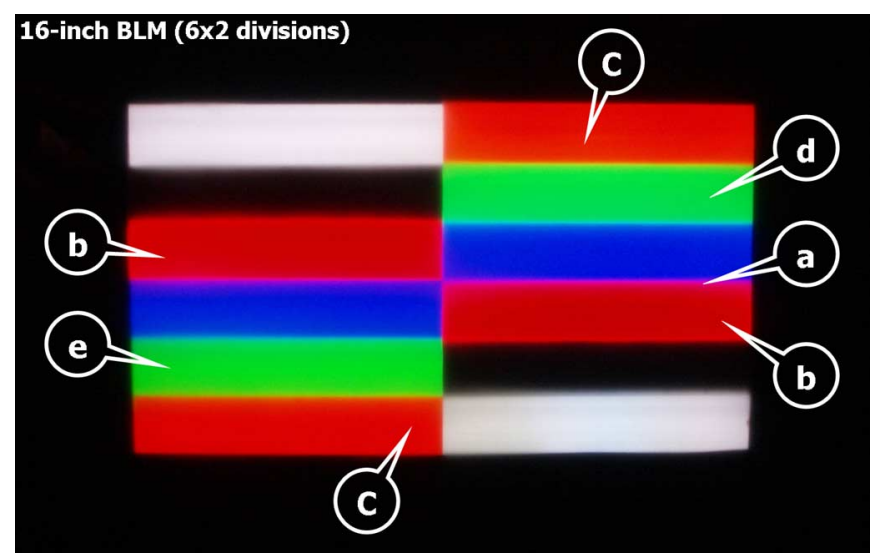

Fig. 8. A striped flag pattern performed by currently finished 16 -in $6 \times 2$ backlight module. The indices a to e indicate defects of erroneous inter-field color mixture constrained by high-haze diffusing sheets and error caused by fabrication and assembly.

Fig. 8 shows a striped flag pattern rendered by currently finished $6 \times 2$ backlight module. In order to avoid the undesirable discontinuity between individual partitions, we employed a high-haze diffusing sheet upon the LGP in the tradeoff between the uniformity and directionality. The optical leakage resulted from the scattering would lead to an unavoidable erroneous inter-field color mixture, as shown in Fig. 8, where a unapparent purple line (marked a) was observed by the red and blue color mixing. Fortunately, such undesirable behavior can be alleviated by changing the sequence order with dual scanning approach [11]. Because the neighboring blocks were dissimilar color, the regions marked $\mathrm{b}$ and $\mathrm{c}$ should be saturated red in original but appeared purplish red and orangey red, respectively. Hence, the penetrated extent of the leaked light has a significant influence on the color exhibition. When pixel values consistent with certain block are being scanned, at least three blocks (itself and both adjacent blocks) must be turned off. In other words, if the leaked light arisen from the illuminated block does not affect beyond a surrounding block in one side, the inter-field erroneous color mixture can be suppressed effectively.

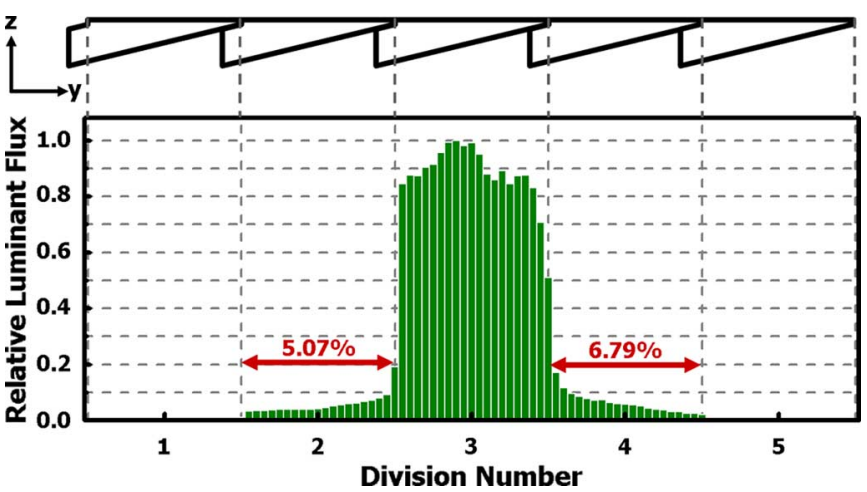

Fig. 9. Measured relative luminant flux between five divisions. In this measurement, only the division number 3 was illuminated to evaluate light leakage. The total leaky flux is down to $11.86 \%$ which causes $5.07 \%$ and $6.79 \%$ in the second and fourth partition, respectively.

In order to evaluate the extent of the light leakage into adjacent unit, the relative luminant flux between five divisions was measured by the conoscope [17] and shown in Fig. 9. In this measurement, only single block (division number 3 ) with $\mathrm{G}$ color was illuminated to investigate the influence of light leakage. The peak luminance was on the centre with $83 \%$ uniformity. The leaky flux into the adjacent partition (the second and fourth LGs) was down to about $5.07 \%$ and $6.79 \%$, respectively. Compared with the simulated leakage, the somewhat different value (11.86\%) in experimental result was due to the error caused by fabrication and assembly. Besides, the amount of the leakage into the forth partitions was slightly larger than that into the second one due to the wedge-shape design. The asymmetric leakage was the reason why the regions marked $\mathrm{d}$ and e (in Fig. 8) appeared cyan(ish) and yellowish but the colors of the adjacent blocks were the same. In addition to the adjacent partitions, the leakage into further partitions of the first and the fifth LGs were extremely low and can be negligible. With field sequential scanning, the optical characteristic of average luminance in $50 \%$ duty cycle can be achieved to 2750 nits. The resulted color gamut was $100.6 \%$ of NTSC standard, and the correlated color temperature of the white point was $7110 \mathrm{~K}$.

\section{CONCLUSION}

In this paper, we demonstrated a region-partitioned LED backlights for a large-sized scanning FSC approach. In addition to the luminous properties such as brightness, directionality, and uniformity, another concerned issue for the FSC approach is the optical isolation within each partitioned unit. Therefore, we proposed a novel optomechanical configuration with edge lighting to alleviate the erroneous inter-field color mixture and reduce the module thickness down to $25 \mathrm{~mm}$ of the overall backlight system. The backlight system consists of a serial of tandem wedge-shaped LGs. Each unit accompanies a set of micro-line-prism over the back surface to control the luminous behavior. Combined with chosen diffusing and prism sheets, the uniformity of one division can be yield to $83 \%$. Undesirable shades or bright lines between boundaries are indistinguishable.

The average luminance of 2750 nits could be achieved by $50 \%$ flashing duration. Consequently, the brightness of the dis- 
play combined with $20 \%$ transmittance of OCB-mode LC panel can be expected up to 550 nits [12]. In addition, the driving current of the R, G, and B LEDs per each light-bar unit were 300, 345 , and $145 \mathrm{~mA}$, respectively, and corresponds the electrical power of $2.034 \mathrm{~W}$. In such case, the total power consumption of the proposed 32-in scanning FSC backlight was about $97.61 \mathrm{~W}$.

The formation of the serially arranged wedge-shaped LG units is able to well control the luminance distribution. The leaky flux from one flashing division was $11.86 \%$ and only influenced upon the adjacent blocks. Minor color mixture raised by the flux leakage can be overcome by the scanning algorithm. The leakage into further partitions of the first and the fifth LGs were extremely low and can be negligible. The proposed tandem type backlight system is an adequate candidate for scanning FSC LCDs. The advantage of modularization can also be scaled up to any other size by modifying the relevant parameters of each mosaic structure accordingly.

\section{ACKNOWLEDGMENT}

The authors would like to thank C.-Y. Lin at ChungHwa Picture Tubes, Ltd. (CPT), Taipei, Taiwan, R.O.C., and Industry Technology Research Institute (ITRI), HsinChu, Taiwan, R.O.C., for their technical assistance of implementation.

\section{REFERENCES}

[1] T. Uchida, "A liquid crystal multicolor display using color filters," in Proc Eurodisplay, 1981, pp. 39-42.

[2] T. Uchida, "Multicolored liquid crystal displays," Opt. Eng, vol. 23, no. 3, pp. 247-252, 1984

[3] T. Uchida, S. Yamamoto, and Y. Shibata, "A full-color matrix liquidcrystal display with color layers on the electrodes," in IDRC Conf. Rec., 1982, pp. 166-170.

[4] K. Sekiya, K. Wako, S. Nakano, T. Ishinabe, T. Miyashita, and T. Uchida, "Overdrive for compensating color-shift on field sequential color TFT-LCDs," in SID Symp. Dig. Tech. Papers, 2004, vol. 35, pp. 408-411.

[5] T. Uchida, K. Saitoh, T. Miyashita, and M. Suzuki, "Field sequential full color LCD without color filter for AM-LCD," in IDRC Conf. Rec., 1997, pp. 37-40.

[6] F. Yamada, H. Nakamura, Y. Sakaguchi, and Y. Taira, and Y. Taira, "Color sequential LCD based on OCB with an LED backlight," in SID Symp. Dig. Tech. Papers, 2000, vol. 31, pp. 1180-1183.

[7] N. Koma, T. Miyashita, T. Uchida, and N. Mitani et al., "Color field sequential LCD using an OCB-TFT-LCD," in SID Symp. Dig. Tech. Papers, 2000, vol. 31, pp. 632-635.

[8] J. Yoo et al., "Side light type field sequential color LCD using divided light guide plates," in IDRC Conf. Rec., 2003, pp. 180-183.

[9] W. C. Tai, S. J. Chiou, C. N. Mo, and C. L. Liu, "Field sequential color LCD-TV using multi-area control algorithm," in Proc IDMC, 2007, pp. 439-442.
[10] K. Käläntär, T. Kishimoto, K. Sekiya, T. Miyashita, and T. Uchida, "Spatial-temporal scanning backlight for color-field sequential optically compensated bend liquid-crystal display," in SID Symp. Dig.Tech. Papers, 2005, vol. 36, pp. 1316-1319.

[11] K. Sekiya, T. Kishimoto, K. Wako, S. Nakano, H. Ishigami, K. Kaelaentaer, K. Shimabukuro, D. Kunioka, T. Miyashita, and T. Uchida, "Spatial-temporal scanning LED backlight for large size field sequential color LCD," in Proc Asia Display/IDW '05, 2005, pp. 1261-1264.

[12] K. Käläntär, T. Kishimoto, K. Sekiya, T. Miyashita, and T. Uchida, "Spatial-temporal scanning backlight mode for field-sequential-color optically-compensated-bend liquid-crystal display," J. Soc. Inf. Display (SID), vol. 14/2, pp. 151-159, 2006

[13] M. C. Chien, C. H. Tien, C. C. Chen, and Y. H. Lu, "Reion-partitioned LED backlight design for field sequential color LCD," in SID Symp. Dig. Tech. Papers, 2007, vol. 38, pp. 441-444.

[14] C. C. Chen, Y. F. Chen, T. T. Liu, C. H. Chen, M. T. Ho, K. H. Chen, and H. P. Shieh, "Spatial-temporal division in field sequential color techinuqe for color filterless LCD," in SID Symp. Dig. Tech. Papers, 2007, vol. 38, pp. 1806-1809.

[15] Everlight Electronic Co., Ltd. , "High power LED," Retrieved Nov. 30, 2007 [Online]. Available: http://www.everlight.com

[16] Y. K. Cheng, S. H. Chung, and J. L. Chern, "Analysis and reduction of dark zone in ultra-thin wedge-plate displays," J. Soc. Inf. Display (SID), vol. 14/9, pp. 813-818, 2006.

[17] CONOSCOPE, ErgoScope, "Autronic-Melchers GmbH: Visual performance measurement solutions," Retrieved Nov. 30, 2007 [Online]. Available: http://www.autronic-melchers.com/index.php?product=29

[18] T. Uchida, S. Yamamoto, and Y. Shibata, "A full-color matrix liquidcrystal display with color layers on the electrodes," IEEE Trans. Electron Devices, vol. ED-30, no. 5, pp. 503-507, May 1983.

Chung-Hao Tien received the B.S. degree in communication engineering and the Ph.D. degree in electro-optical engineering from National Chiao Tung University, Hsinchu, Taiwan, R.O.C., in 1997 and 2003, respectively.

He joined National Chiao Tung University as an assistant professor at Department of Photonics and Display Institute since 2004 after as a post doctoral research staff at Carnegie Mellon University, Pittsburgh, PA. His current research interests are in optical data storage and non-imaging optics.

Yen-Hsing Lu received the B.S. degree in communication engineering from National Chiao Tung University, Hsinchu, Taiwan, R.O.C., in 2004, and is currently working toward the Ph.D. degree in the Institute of Electro-Optical Engineering, National Chiao Tung University.

His current researches are the optical design for high-dynamic-range display and the color appearance model for LCD.

Yuan-Jung Yao received the B.S. degree in electronic engineering from National Taiwan University of Science Technology, Taiwan, R.O.C., in 2005, and the M.S. degree in Industrial Technology R\&D Master Program on Photonics and Display Technologies from National Chiao Tung University, Taiwan, R.O.C.,in 2007.

Since 2007, he has been an engineer with AU Optronics, Hsinchu Taiwan, R.O.C. His recently research is the optical design of LED package and TFT-LCD backlight module. 